\title{
A Aplicação de Técnicas Isotópicas ao Estudo de Fugas na Barragem de Tapacurá, Pernambuco
}

\author{
Heldio Pereira Villar \\ Centro Regional de Ciências Nucleares e Escola Politécnica da Universidade de Pernambuco \\ R. Cônego Barata, 999 - Tamarineira - CEP 52110-120 Recife, PE - mnogue@nlink.com.br \\ Ricardo de Andrade Lima \\ Centro Regional de Ciências Nucleares \\ R. Cônego Barata, 999 - Tamarineira - CEP 52110-120 Recife, PE - ral@elogica.com.br \\ Antônio Celso Dantas Antonino \\ Departamento de Energia Nuclear da Universidade Federal de Pernambuco \\ Av. Prof. Luiz Freire, 1000 - Cid. Universitária - CEP 54740-540 Recife, PE - acda@npd.ufpe.br
}

Marco Otávio Alencar Menezes

Escola Politécnica da Universidade de Pernambuco

Pça. do Internacional, 455 - Madalena - CEP 50750-470 Recife, PE

\section{Carlos Alberto Brayner de Oliveira Lira}

Departamento de Energia Nuclear - UFPE

Av. Prof. Luiz Freire, 1000 - Cid. Universitária - CEP 54740-540 Recife, PE - cabol@npd.ufpe.br

\author{
Affonso Augusto Gusmão Vianna \\ Companhia Pernambucana de Saneamento \\ R. da Aurora, 777 - Boa Vista - CEP 50060-010 Recife, PE \\ Jefferson Viana Bandeira \\ Centro de Desenvolvimento da Tecnologia Nuclear \\ R. Prof. Mário Werneck, s/n - Cid. Universitária - CEP 30123-970 Belo Horizonte, MG \\ Recebido: 25/01/00 - revisão: 14/08/00 - aceito: 10/05/01
}

\section{RESUMO}

Um estudo de possíveis fugas sob o maciço de concreto da barragem de Tapacurá (PE) foi realizado por meio de técnicas isotópicas baseadas em isótopos estáveis ambientais. Para isso, é feita a comparação entre as concentrações de isótopos estáveis (deutério, ${ }^{18} \mathrm{O} e{ }^{13} \mathrm{C}$ ) nas amostras de água coletadas a montante e a jusante da barragem. A análise dos resultados encontrados mostrou que a água a montante da barragem tinha uma origem diferente daquela coletada a jusante, indicando dessa forma a inexistência de fuga através da formação geológica da fundação da barragem.

Palavras-chave: fuga; barragem; isótopos.

\section{INTRODUÇÃO}

Concluída em julho de 1973, a Barragem de Tapacurá é uma obra do governo federal destinada a abastecer $40 \%$ da Região Metropolitana do Recife (o que corresponde a cerca de 1.200 .000 pessoas) e, paralelamente, controlar as enchentes periódicas que assolaram a capital de Pernambuco por várias décadas. A barragem em si é parte de um sistema compreendendo uma barragem principal, de con- creto, e duas barragens auxiliares, de terra, como mostra a Figura 1.

Esse sistema de barragens foi construído sobre o rio Tapacurá, no município de São Lourenço da Mata, cerca de $40 \mathrm{~km}$ a oeste do Recife. As características físicas do sistema são mostradas na Tabela 1.

Uma década após ter sido comissionada, a barragem de concreto passou a apresentar sensível percolação de água, tanto pelo sistema de drena- 


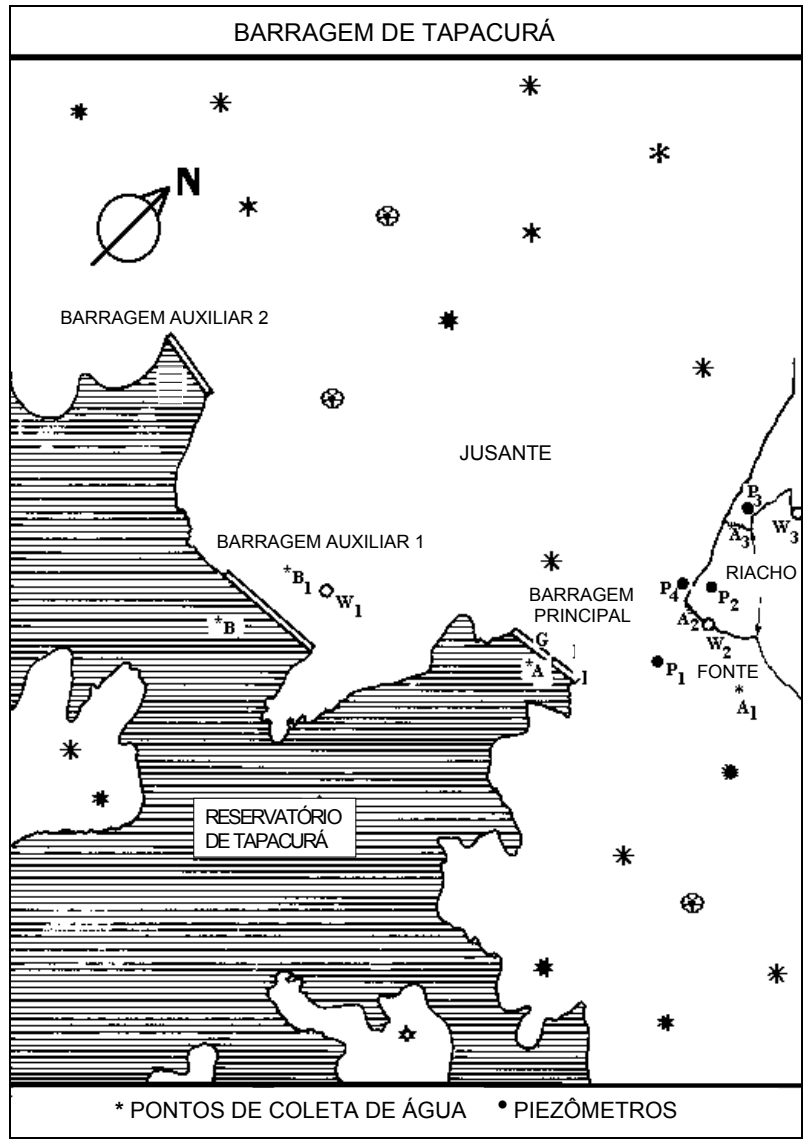

Figura 1. Mapa do sistema de barragens do rio Tapacurá, mostrando pontos de coleta. Escala - 1: 25.000.

gem como através de fissuras no maciço de concreto. $\mathrm{O}$ risco a que estariam expostas as populosas comunidades a jusante da barragem, levou os governos federal e estadual a procurar meios para recuperar estruturalmente a barragem e reduzir os vazamentos. Isso foi feito, por intermédio de convênios, a partir de 1992.

Foi então contratada uma consultoria especializada em recuperação de estruturas para analisar o problema, propor soluções e fiscalizar a execução dos serviços no maciço de concreto. Instrumentos para monitorar a estabilidade da barragem foram instalados. Em alguns meses foram eliminados quase todos os vazamentos (restando apenas os de pequena monta). Em seu relatório, porém, os consultores recomendaram que uma investigação sobre possíveis fugas através da formação geológica sob a barragem fosse realizada.

Essa formação, um migmatito Precambriano de composição grano-diorítica próximo ao lineamento de Pernambuco (zona de cisalhamento), apresenta foliações (mergulho $215^{\circ} \mathrm{Az}$ ) e fraturas.
Tabela 1. Características físicas do sistema Tapacurá.

\begin{tabular}{lc}
\hline Característica & Valor \\
\hline $\begin{array}{l}\text { Comprimento total da barragem de } \\
\text { concreto; }\end{array}$ & $252 \mathrm{~m}$ \\
$\begin{array}{l}\text { Comprimento total do dique fusível } \\
\text { em terra; }\end{array}$ & $128 \mathrm{~m}$ \\
$\begin{array}{l}\text { Altura máxima da barragem de } \\
\text { concreto; }\end{array}$ & $35 \mathrm{~m}$ \\
$\begin{array}{l}\text { Largura do coroamento da barragem } \\
\text { de concreto (trecho não-vertedor); }\end{array}$ & $4,5 \mathrm{~m}$ \\
$\begin{array}{l}\text { Volume de concreto; } \\
\text { Volume armazenado na cota máxima } \\
\text { (110 m acima do nível do mar); }\end{array}$ & $170 \times 10^{6} \mathrm{~m}^{3}$ \\
$\begin{array}{l}\text { Volume armazenado na cota do } \\
\text { vertedor central (cota 103 m); }\end{array}$ & $95 \times 10^{6} \mathrm{~m}^{3}$ \\
$\begin{array}{l}\text { Área máxima do reservatório; } \\
\text { Área do reservatório na cota 103 m; }\end{array}$ & $1300 \mathrm{ha}^{3}$ \\
$\begin{array}{l}\text { Descarga máxima regularizada; } \\
\text { Capacidade máxima de fornecimento }\end{array}$ & $20 \times 1 \mathrm{~m}^{3} / \mathrm{s}$ \\
\hline
\end{tabular}

As fraturas são comumente sub-verticais ou subhorizontais, sendo as primeiras parcialmente preenchidas com rochas intrusivas. São tipicamente observadas 4 a 8 fraturas por metro, o que favorece a percolação da água, mormente nas seções onde o intemperismo é mais acentuado. O solo residual na região de Tapacurá apresenta quase sempre uma espessura inferior a $3 \mathrm{~m}$.

O presente trabalho tem por objetivo o estudo de fugas na barragem de Tapacurá, PE, por meio de técnicas isotópicas.

\section{MATERIAIS E MÉTODOS}

\section{A análise isotópica em hidrologia}

É sabido que diversos processos ambientais (evaporação, condensação, reações físico-químicas) levam ao fracionamento de um elemento em seus isótopos constituintes. Como resultado, cada elemento apresenta uma "assinatura" característica dos processos que ele teve de atravessar. Isso acontece, por exemplo, com os elementos que compõem a água, não apenas o hidrogênio e o oxigênio, mas também aqueles que entram na composição dos sais e dos outros compostos nela dissolvidos.

Na prática da hidrologia isotópica, as diferentes concentrações dos isótopos de interesse para a investigação são expressas na forma de um valor $\delta$, definido, para cada isótopo, como o desvio em \%o 
relativo a um padrão denominado SMOW (Standard Mean Ocean Water, ou Padrão Médio da Água do Mar) (Domenico e Schwartz, 1990; Faure, 1986).

Os isótopos mais comumente empregados em hidrologia são o deutério $\left(\mathrm{D}\right.$, ou $\left.{ }^{2} \mathrm{H}\right), \mathrm{o}^{18} \mathrm{O}$ e o ${ }^{13} \mathrm{C}$. As concentrações desses isótopos em uma amostra são usualmente determinadas por espectrometria de massa, sendo a seguir o valor $\delta$ calculado segundo (Bowen, 1994):

$$
\delta=\frac{\mathrm{C}_{\text {amostra }}-\mathrm{C}_{\mathrm{SMOW}}}{\mathrm{C}_{\mathrm{SMOW}}} \times 1000
$$

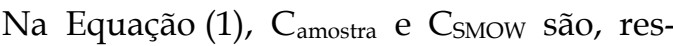
pectivamente, a concentração do isótopo escolhido na amostra e no padrão médio da água do mar. A análise por espectrometria de massa apresenta uma exatidão de $\pm 2 \%$ para o deutério e de $\pm 0,2 \%$ o para o ${ }^{18} \mathrm{O}$.

\section{A espectrometria de massa}

O emprego da análise isotópica em hidrologia e outras aplicações está condicionado à identificação e quantificação dos isótopos componentes de um certo elemento químico. Como o comportamento químico dos isótopos de um mesmo elemento é virtualmente idêntico (posto que esse comportamento está condicionado à estrutura da última camada de eléctrons, que é a mesma para todos os isótopos de cada elemento), investigações acerca da presença e quantidade desses isótopos não podem ser feitas pelas técnicas químicas convencionais, como a espectrofotometria de absorção atômica ou a cromatografia.

A grande diferença entre dois isótopos de um mesmo elemento está em suas massas atômicas. Assim sendo, o processo óbvio para separação de isótopos deve levar em conta essa diferença de massa. $\mathrm{O}$ diferente comportamento de átomos de massas diferentes é enfatizado num instrumento idealizado há mais de três quartos de século, o espectrômetro de massa (Kaplan, 1956; Oldenberg e Holladay, 1971).

O funcionamento do espectrômetro de massa se baseia no princípio da força magnética que é exercida sobre uma carga em movimento, indicada na Figura 2 (Romer, 1964).

Essa força $F_{m}$ tem módulo dado por:

$$
F_{m}=q v B \operatorname{sen} \theta
$$

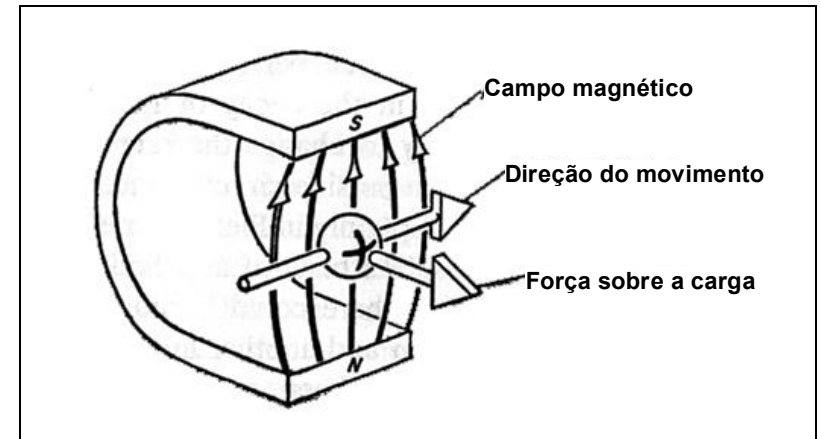

Figura 2. Força magnética atuante sobre uma carga positiva móvel, provocada pela aplicação de um campo magnético perpendicular à direção do deslocamento.

onde q é a magnitude da carga, $v$ sua velocidade e B a magnitude do campo magnético atuante, cujas linhas de força formam um ângulo $\theta$ com a direção do deslocamento da carga. A força $F_{m}$ é perpendicular tanto ao campo magnético quanto à direção do deslocamento. Dessa forma, caso o campo magnético seja dirigido perpendicularmente ao deslocamento da carga, esta executará uma trajetória circular, com a força magnética fazendo o papel de força centrípeta. Logo, o raio dessa trajetória circular é calculado segundo (Kaplan, 1956; Tipler, 1995):

$$
\mathrm{F}_{\mathrm{m}}=\mathrm{qvB} \operatorname{sen} \frac{\pi}{2}=\mathrm{m} \frac{\mathrm{v}^{2}}{\mathrm{r}} \therefore \mathrm{r}=\frac{\mathrm{mv}}{\mathrm{qB}}
$$

Fica evidenciado, a partir da Equação (3), que o raio de curvatura de diversas cargas de igual magnitude e velocidade, sujeitas ao mesmo campo magnético, irá variar linearmente com cada massa. O espectrômetro de massa é um aparelho no qual átomos são ionizados (adquirem carga elétrica positiva por meio de processos físicos que extraem desses átomos um eléctron), são acelerados em um campo elétrico até que atinjam uma certa velocidade, entrando finalmente em uma câmara evacuada onde atua um campo magnético perpendicular ao seu deslocamento. As trajetórias dos íons são encurvadas segundo suas massas e eles são a seguir coletados por detectores de íons. A posição em que um íon é detectado é função de sua massa. A quantificação dos íons de um elemento permite então que se estabeleça sua composição isotópica.

\section{Coleta e tratamento das amostras}

Os pontos de coleta de amostras estão indicados na Figura 1. A Tabela 2 descreve sumariamente as características desses pontos. 
Tabela 2. Características dos pontos de coleta de amostras.

\begin{tabular}{|c|c|}
\hline Ponto & Descrição \\
\hline $\mathrm{A}, \mathrm{B}$ & Reservatório; \\
\hline $\mathrm{A} 1, \mathrm{~A} 2, \mathrm{~A} 3$ & $\begin{array}{l}\text { Fontes e córregos a jusante da } \\
\text { barragem principal; }\end{array}$ \\
\hline B1 & $\begin{array}{l}\text { Fonte a jusante da barragem } \\
\text { auxiliar, próximo à parede de } \\
\text { concreto; }\end{array}$ \\
\hline G & Galeria da barragem principal; \\
\hline $\mathrm{P} 1, \mathrm{P} 2, \mathrm{P} 3, \mathrm{P} 4$ & $\begin{array}{l}\text { Piezômetros construídos a jusante } \\
\text { da barragem principal. }\end{array}$ \\
\hline
\end{tabular}

As coletas foram realizadas ao longo de um período de 20 meses, de maneira que todas as estações do ano foram cobertas. Em conseqüência, a repetição de coletas deixou de ser realizada nos pontos A3 e B1, em virtude da baixa precipitação observada na estação seca. Ao mesmo tempo, como o progresso da investigação apontava para uma grande semelhança nas características das amostras retiradas dos pontos A e B (dentro do reservatório), as amostras de água do reservatório ficaram restritas às coletadas no ponto $\mathrm{A}$.

Cada amostra era acondicionada em um recipiente estéril de plástico. Para as determinações relativas ao teor de ${ }^{13} \mathrm{C}$, esses recipientes continham uma solução de $\mathrm{NaOH}$ de $\mathrm{pH} 12$ à qual era adicionada uma solução de $\mathrm{BrCl}_{2}$ (ou $\mathrm{SrCl}_{2}$ ) a uma concentração de $3 \mathrm{~g} / \mathrm{L}$. Obtinha-se dessa forma um precipitado, que era retirado com uma mangueira no próprio local de coleta.

Todas as análises isotópicas para o ${ }^{13} \mathrm{C}$ foram realizadas em seis amostras nos laboratórios da Agência Internacional de Energia Atômica (IAEA) em Viena, Áustria. As análises de deutério e $18 \mathrm{O}$ foram realizadas em onze amostras nos laboratórios do Centro de Estudos e Experimentação de Obras Públicas (CEDEX) em Madri, Espanha.

\section{RESULTADOS}

\section{Deutério $\left({ }^{2} \mathrm{H}\right)$ e oxigênio-18 $(18 \mathrm{O})$}

A Tabela 3 mostra os resultados obtidos para o valor $\delta$ para o deutério e o oxigênio-18. Os valores são apresentados em \%o.

Como os pontos A e B ficam dentro do reservatório e o ponto $G$ é na galeria da barragem principal, de maneira que a água aí existente resul-
Tabela 3. Análise isotópica - Valor $\delta(\mathrm{em} \%)$ para deutério e oxigênio-18.

\begin{tabular}{crr}
\hline Amostra & $\delta \mathrm{D}$ & $\delta^{18} \mathrm{O}$ \\
\hline A & 5,30 & 0,10 \\
A1 & $-2,89$ & $-2,00$ \\
A2 & 0,79 & $-1,40$ \\
A3 & 1,54 & $-1,60$ \\
B & 4,90 & 0,20 \\
B1 & 1,71 & $-1,50$ \\
G & 4,74 & 0,08 \\
P1 & 0,07 & $-1,50$ \\
P2 & $-0,82$ & $-1,40$ \\
P3 & $-13,30$ & $-3,21$ \\
P4 & $-7,18$ & $-2,60$ \\
\hline
\end{tabular}

ta da percolação da água do reservatório através do maciço de concreto da barragem, foi determinada, com os resultados das amostras desses três pontos a média do valor $\delta$ para o deutério e para o ${ }^{18} \mathrm{O}$ na água do reservatório. Foi encontrado um $\delta \mathrm{D}$ igual a $4,98 \%$ e um $\delta^{18} \mathrm{O}$ igual a $0,12 \%$. As discrepâncias encontradas nos resultados dessas amostras, tanto para o deutério como para o ${ }^{18} \mathrm{O}$, estão dentro das incertezas analíticas esperadas.

Os resultados acima foram superpostos à chamada "linha meteórica", que descreve a relação entre $\delta \mathrm{D}$ e $\delta^{18} \mathrm{O}$ para amostras ambientais. Essa comparação está mostrada na Figura 3.

Verifica-se de maneira inequívoca a discrepância entre as relações $\delta \mathrm{D} / \delta^{18} \mathrm{O}$ para as amostras do reservatório e as dos outros pontos de coleta, o que sugere que a origem da água nesses pontos difere daquela do reservatório.

\section{Carbono-13 $\left({ }^{13} \mathrm{C}\right)$}

Os estudos relativos à concentração do isótopo ${ }^{13} \mathrm{C}$ em águas visam principalmente estabelecer a origem dos solutos carbonatados presentes (Domenico e Schwartz, 1990). Um valor típico em águas superficiais para o $\delta\left({ }^{13} \mathrm{C}\right)$ é de $-8,5 \%$. Os resultados obtidos para as seis amostras analisadas estão mostrados na Tabela 4.

Como se pode observar, os valores para as amostras dos pontos A e G (que são de água do reservatório), são bastante similares entre si e ao valor médio considerado para águas superficiais. As quatro outras amostras apresentaram um $\delta^{13} \mathrm{C}$ bem mais negativo, o que indica que o radical 


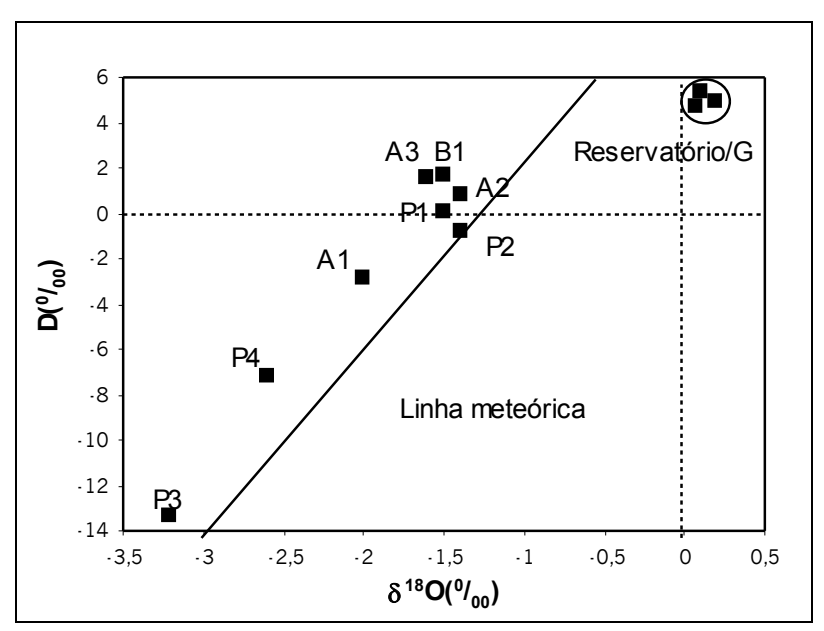

Figura 3. Comparação entre as relações $\delta \mathrm{D} / \delta^{18} \mathrm{O}$ para diversas amostras e a linha meteórica.

Tabela 4. Análise isotópica - Valor $\delta(e m \%)$ para ${ }^{13} \mathrm{C}$.

\begin{tabular}{cr}
\hline Amostra & $\delta^{13} \mathrm{C}$ \\
\hline A & $-8,94$ \\
A1 & $-18,18$ \\
G & $-9,59$ \\
P1 & $-17,30$ \\
P2 & $-14,02$ \\
P3 & $-15,16$ \\
\hline
\end{tabular}

$\mathrm{HCO}_{3}$ - dissolvido é biogênico e não devido ao equilíbrio com o gás carbônico presente na atmosfera, como aparentemente é o caso para as outras duas amostras. Fica, assim, confirmada a hipótese pela qual a água dos córregos, fontes e piezômetros a jusante da barragem principal não é a mesma do reservatório.

\section{CONCLUSÕES}

- A origem da água a montante da barragem foi diferente daquela coletada a jusante, indicando dessa forma a inexistência de fuga através da formação geológica da fundação da barragem.

- Embora tenha essa sido a primeira aplicação da análise de isótopos estáveis em hidrologia na Região Nordeste do Brasil, parece evidenciada a viabilidade de seu emprego em estudos de fugas de barragens.

- A técnica se mostrou suficientemente precisa para permitir também a determinação da origem de águas subterrâneas; numa região de escassos recursos hídricos, isso pode ser de fundamental importância para a outorga de mananciais.

\section{AGRADECIMENTOS}

O presente trabalho foi realizado dentro do Programa ARCAL (Arreglos Regionales Cooperativos para la Promoción de la Ciencia y la Tecnologia Nucleares en America Latina) XVIII da Agência Internacional de Energia Atômica, ao qual os autores agradecem o importante apoio recebido.

\section{REFERÊNCIAS}

BOWEN, R. (1994). Isotopes in the Earth Sciences, Chapman \& Hall, Londres, $647 \mathrm{p}$.

DOMENICO, P. A. e SCHWARTZ, F. W. (1990). Physical and Chemical Hydrogeology, Wiley, New York, 842 p.

FAURE, G. (1986). Principles of Isotope Geology, Wiley, New York, 589 p.

KAPLAN, I. (1956). Nuclear Physics, Addison-Wesley, Reading, $770 \mathrm{p}$.

OLDENBERG, O. e HOLLADAY, W. (1971). Introdução à Física Atômica e Nuclear, Edgard Blücher, São Paulo, $371 \mathrm{p}$.

ROMER, A. (1964). The Restless Atom, Dover, New York, $198 \mathrm{p}$.

TIPLER, P. (1995). Física - volume 3, Guanabara Koogan, Rio de Janeiro, $340 \mathrm{p}$.

The Application of Isotopic Techniques to the Investigation of Leaks at the Tapacurá Dam, Pernambuco

\section{ABSTRACT}

An investigation of the possible leaks under Tapacurá Dam's main concrete body was carried out using isotopic techniques based on environmental isotopes. For this purpose, concentrations of stable isotopes (deuterium, ${ }^{18} \mathrm{O}$ and ${ }^{13} \mathrm{C}$ ) in samples collected upstream and downstream from the dam were compared. The analysis of results showed that water upstream and downstream from the dam came from different sources. This fact suggested that there were no leaks through the geological formation under the dam's foundations.

Key-words: leaks; dam; isotopes. 